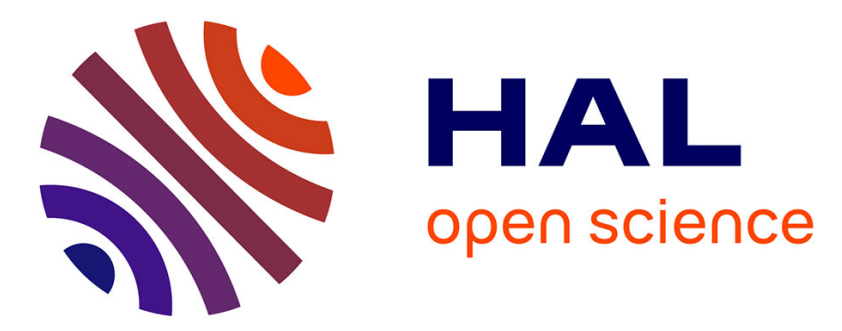

\title{
Simulation of surface waves generated by an underwater landslide in a bounded reservoir
}

Sonya Beizel, Leonid Chubarov, Denys Dutykh, Gayaz Khakimzyanov, Nina Shokina

\section{- To cite this version:}

Sonya Beizel, Leonid Chubarov, Denys Dutykh, Gayaz Khakimzyanov, Nina Shokina. Simulation of surface waves generated by an underwater landslide in a bounded reservoir. Russ. J. Num. Anal. Math. Modelling, 2012, 27 (6), pp.539-558. 10.1515/rnam-2012-0031 . hal-00788284v2

\section{HAL Id: hal-00788284 \\ https://hal.science/hal-00788284v2}

Submitted on 29 May 2020

HAL is a multi-disciplinary open access archive for the deposit and dissemination of scientific research documents, whether they are published or not. The documents may come from teaching and research institutions in France or abroad, or from public or private research centers.
L'archive ouverte pluridisciplinaire HAL, est destinée au dépôt et à la diffusion de documents scientifiques de niveau recherche, publiés ou non, émanant des établissements d'enseignement et de recherche français ou étrangers, des laboratoires publics ou privés.

\section{(ㅇ)(1) 80}

Distributed under a Creative Commons Attribution - NonCommercial - ShareAlikel 4.0 


\title{
Simulation of surface waves generated by an underwater landslide in a bounded reservoir
}

\author{
S. A. BEIZEL ${ }^{*}$, L.B.CHUBAROV*, D. DUTYKH*，G.S.KHAKIMZYANOV, \\ and N. Yu. SHOKINA*
}

\begin{abstract}
Equations of a landslide motion over an uneven underwater slope subject to gravity and buoyancy forces, water friction and resistance are presented. A simulation of surface waves generated by a landslide in a bounded reservoir with a parabolic bottom profile has been performed within the nonlinear shallow water equations, and the results of that simulation are given. The influence of the parameters of the motion equation on the maximal splashing size is studied numerically.
\end{abstract}

The importance of taking into account the landslide mechanism of surface wave generation in studies of catastrophic events in water areas of various scales is beyond any doubt $[1,13]$. Such mechanism occurred, for example, in the generation of the tsunami near Papua New Guinea (1998). There are conjectures among the experts that the landslide effects had a considerable impact on the formation of megatsunami in 2004 (Sumatra) and in 2011 (Tohoku, Japan).

Some attempts at experimental study of surface waves generated in the motion of a rigid landslide model over a flat underwater slope have been undertaken recently $[6,9-11,20]$. A series of papers considered numerical simulations of such problems (see, e.g., $[3,9,16,19,20])$ using the law describing the motion of a rigid landslide over a flat slope $[12,15,20]$.

Naturally, modelling of real events has to take into account the underwater slope unevenness. Thus, in [5], an analytic solution to the linear shallow water equations was obtained for two special forms of an uneven slope under the landslide. The results of numerical simulation of a landslide motion over an uneven slope consisting of two flat slopes were described in [18]. In paper [4], an equation was proposed for a landslide motion over an uneven slope subject to the forces of gravity, buoyancy, and water resistance. Numerical investigation of particular cases [2] has shown that the pattern of the surface waves in the motion of a landslide over an uneven bottom essentially differs from that in the case of a flat slope.

The derivation of the landslide motion equation presented in [4] assumes that the

\footnotetext{
* Institute of Computational Technologies, Siberian Branch of the Russian Academy of Sciences, Novosibirsk 630090, Russia

The work was supported by the Russian Foundation for Basic Research (project 10-05-91052NTsNI), by the Program of Integration Research, Siberian Branch of RAS (project 42), and by the Grant Council of the President of Russian Federation for state support of leading scientific schools of RF (project NSh-6293.2012.9).
} 
landslide and the underwater slope are 'one-dimensional', i.e., their form depends only on one spatial coordinate and is constant along the second spatial direction. In this paper we present the derivation of equations in the general case of the motion of a spatially nonuniform underwater landslide over a spatially nonuniform underwater slope. In this case the landslide is represented by a 'quasi-rigid' volume of a continuous medium whose surface form keeps changing according to the slope unevenness encountered in the course of the movement (as a deformable body), whereas the horizontal components of the velocity vector are the same at each point of the landslide (as in a rigid body under translational movement).

We also present the results of numerical experiments for a bounded water reservoir with a parabolic form of the bottom. In those experiments we have used the shallow water model to study the dependence of the characteristics of the generated waves on the coefficients of friction, resistance, and associated mass, on the density of the landslide material and its size.

\section{Landslide motion equations}

We consider a liquid layer bounded from above by a free surface $z=\eta(x, y, t)$ and from below by a movable impermeable boundary $z=-h(x, y, t)=h_{\mathrm{bt}}(x, y)+$ $h_{\mathrm{sl}}(x, y, t)$, where $t$ is the time and the coordinate system $O x y z$ is chosen so that $x$ and $y$ are the horizontal coordinates, $z$ is the vertical one; in this case the plane $z=0$ coincides with the surface of the resting fluid. The mobility of the bottom boundary is caused by the fact that the landslide, whose form is described by a nonnegative bounded function $z=h_{\mathrm{sl}}(x, y, t)$, keeps moving over the impermeable bottom given by the single-valued function

$$
z=h_{\mathrm{bt}}(x, y) \text {. }
$$

Assume that at the initial time moment $t=0$ the landslide and the fluid are in their rest states and the function $z=h_{\mathrm{sl}}^{0}(x, y)$ with a finite support $\mathscr{D}_{0}$ describing the initial form of the landslide is given so that $h_{\mathrm{sl}}(x, y, 0)=h_{\mathrm{sl}}^{0}(x, y)$. The form and the position of the landslide is determined for $t>0$ by the law of its motion. Deriving the motion equations, we identify the landslide with a material point moving over a curved surface. However, the force acting onto this point is calculated as an integral sum of all forces acting onto the elementary volumes of the landslide. Thus, we take into account the form of the landslide and its position on the uneven slope in the calculation of the forces.

Therefore, we assume that the position of the landslide at each time moment is determined by a certain point $\boldsymbol{x}_{c}(t)=\left(x_{c}(t), y_{c}(t), z_{c}(t)\right)$ sliding along uneven bottom (1.1) according to the law of non-free motion of a material point over a given surface

$$
M \ddot{x}_{c}=F_{1}, \quad M \ddot{y}_{c}=F_{2}, \quad M \ddot{z}_{c}=F_{3}
$$

in this case

$$
z_{c}(t)=h_{\mathrm{bt}}\left(x_{c}(t), y_{c}(t)\right)
$$


where $x_{c}(0)=x_{c}^{0}, y_{c}(0)=y_{c}^{0},\left(x_{c}^{0}, y_{c}^{0}\right) \in \mathscr{D}_{0}, z_{c}(0)=z_{c}^{0}=h_{\mathrm{bt}}\left(x_{c}^{0}, y_{c}^{0}\right), F_{1}, F_{2}, F_{3}$ are the components of the force vector $\boldsymbol{F}$ which we define later, $M=\left(\rho_{\mathrm{sl}}+C_{w} \rho_{w}\right) V$ is the landslide mass together with the associated mass of water, $\rho_{w}$ is the water density, $\rho_{\mathrm{sl}}$ is the landslide density $\left(\rho_{\mathrm{sl}}>\rho_{w}\right), C_{w}$ is the coefficient of the associated mass, $V$ is the landslide volume. The coordinates $x_{c}(t), y_{c}(t)$ can be taken as the corresponding coordinates of the center of the landslide mass.

Below we assume that for $t>0$ the surface of the landslide is described by the function $z=h_{\mathrm{bt}}(x, y)+h_{\mathrm{sl}}(x, y, t)$, where

$$
h_{\mathrm{sl}}(x, y, t)=h_{\mathrm{sl}}^{0}\left(x+x_{c}^{0}-x_{c}(t), y+y_{c}^{0}-y_{c}(t)\right) .
$$

Thus, the function $h_{\mathrm{sl}}(x, y, t)$ is finite and its support $\mathscr{D}_{t}$ is completely determined by the support of the function $h_{\mathrm{sl}}^{0}$ and the position of the point $\boldsymbol{x}_{c}(t)$ :

$$
\mathscr{D}_{t}=\left\{(x, y) \mid\left(x+x_{c}^{0}-x_{c}(t), y+y_{c}^{0}-y_{c}(t)\right) \in \mathscr{D}_{0}\right\} .
$$

Note some peculiarities of the proposed physical model of the landslide process. Formula (1.5) implies that at each time moment $t$ the landslide is positioned on the slope so that its projection onto the plane $z=0$ coincides with the set $\mathscr{D}_{t}$, which is obtained by translation of the set $\mathscr{D}_{0}$. This means that all points of the landslide have the same instant horizontal velocity. However, different points of the landslide have different velocities in the vertical direction, and the area of the contact of the landslide with the bottom changes in time. The landslide is stretched out on steep sections and is shortened on gentle ones (along the slope), which reflects the real situation to some extent. Thus, when the landslide is moving, its surface is deformed according to the bottom unevenness and, therefore, we say that the motion of a real landslide is simulated by the motion of a quasi-rigid body. For a flat slope, this approach gives not only the same horizontal velocities for all points of the landslide, but also equal vertical components, i.e., the landslide moves over a flat slope as a rigid body. It is interesting that the volume of the landslide remains constant despite its deformation:

$$
V=\iint_{\mathscr{D}_{t}} h_{\mathrm{sl}}(x, y, t) \mathrm{d} x \mathrm{~d} y=\iint_{\mathscr{D}_{0}} h_{\mathrm{sl}}^{0}(x, y) \mathrm{d} x \mathrm{~d} y=\text { const. }
$$

Deriving the landslide motion equations, we assume that surface (1.1) has no singular points and admits the regular parameterization

$$
x=x\left(q^{1}, q^{2}\right), \quad y=y\left(q^{1}, q^{2}\right), \quad z=z\left(q^{1}, q^{2}\right)
$$

where $q^{1}$ and $q^{2}$ are the parameters. Then

$$
x_{c}(t)=x\left(q^{1}(t), q^{2}(t)\right), \quad y_{c}(t)=y\left(q^{1}(t), q^{2}(t)\right), \quad z_{c}(t)=z\left(q^{1}(t), q^{2}(t)\right) .
$$


In this case $\left(q^{1}(t), q^{2}(t)\right)$ is the position of the point $\boldsymbol{x}_{c}$ in the parametric space at the time moment $t$. Parameterization relations (1.7) imply the following expressions for the Cartesian velocity components through its contravariant components:

$$
\dot{x}_{c}=x_{q^{1}} \dot{q}^{1}+x_{q^{2}} \dot{q}^{2}, \quad \dot{y}_{c}=y_{q^{1}} \dot{q}^{1}+y_{q^{2}} \dot{q}^{2}, \quad \dot{z}_{c}=z_{q^{1}} \dot{q}^{1}+z_{q^{2}} \dot{q}^{2} .
$$

Therefore, motion equations (1.2) can be rewritten as

$$
M \frac{\mathrm{d}}{\mathrm{d} t}\left(x_{q^{1}} \dot{q}^{1}+x_{q^{2}} \dot{q}^{2}\right)=F_{1}, M \frac{\mathrm{d}}{\mathrm{d} t}\left(y_{q^{1}} \dot{q}^{1}+y_{q^{2}} \dot{q}^{2}\right)=F_{2}, M \frac{\mathrm{d}}{\mathrm{d} t}\left(z_{q^{1}} \dot{q}^{1}+z_{q^{2}} \dot{q}^{2}\right)=F_{3} .
$$

Multiplying each equation (1.8) by the corresponding component $x_{q^{1}}, y_{q^{1}}$, or $z_{q^{1}}$ of the vector tangent to surface (1.7) given parametrically and summing the results, we get the first motion equation

$M \frac{\mathrm{d}}{\mathrm{d} t}\left(g_{11} \dot{q}^{1}+g_{12} \dot{q}^{2}\right)-\frac{M}{2}\left[\left(g_{11}\right)_{q^{1}}\left(\dot{q}^{1}\right)^{2}+2\left(g_{12}\right)_{q^{1}} \dot{q}^{1} \dot{q}^{2}+\left(g_{22}\right)_{q^{1}}\left(\dot{q}^{2}\right)^{2}\right]=F_{\tau_{1}} \sqrt{g_{11}}$

where $g_{\alpha \beta}(\alpha, \beta=1,2)$ are the covariant components of the metric tensor of surface (1.1),

$$
\begin{array}{ll}
g_{11}=x_{q^{1}}^{2}+y_{q^{1}}^{2}+z_{q^{1}}^{2}, & g_{12}=g_{21}=x_{q^{1}} x_{q^{2}}+y_{q^{1}} y_{q^{2}}+z_{q^{1}} z_{q^{2}} \\
g_{22}=x_{q^{2}}^{2}+y_{q^{2}}^{2}+z_{q^{2}}^{2}, & F_{\tau_{1}}=\boldsymbol{F} \cdot \boldsymbol{\tau}_{1}
\end{array}
$$

and $\tau_{\alpha}$ are the unit vectors tangent to surface (1.7),

$$
\boldsymbol{\tau}_{\alpha}=\frac{1}{\sqrt{g_{\alpha \alpha}}}\left(x_{q^{\alpha}}, y_{q^{\alpha}}, z_{q^{\alpha}}\right)^{T}, \quad \alpha=1,2 .
$$

The second motion equation

$M \frac{\mathrm{d}}{\mathrm{d} t}\left(g_{21} \dot{q}^{1}+g_{22} \dot{q}^{2}\right)-\frac{M}{2}\left[\left(g_{11}\right)_{q^{2}}\left(\dot{q}^{1}\right)^{2}+2\left(g_{21}\right)_{q^{2}} \dot{q}^{1} \dot{q}^{2}+\left(g_{22}\right)_{q^{2}}\left(\dot{q}^{2}\right)^{2}\right]=F_{\tau_{2} \sqrt{g_{22}}}$

is obtained similarly, here $F_{\tau_{2}}=\boldsymbol{F} \cdot \boldsymbol{\tau}_{2}$.

Now we reduce equations (1.9), (1.11) to a form convenient for numerical integration. Let $v_{1}, v_{2}$ be the covariant components of the velocity vector, $v^{1}=\dot{q}^{1}$, $v^{2}=\dot{q}^{2}$ be the contravariant components. We have

$$
\begin{array}{ll}
v_{1}=g_{11} v^{1}+g_{12} v^{2}, & v_{2}=g_{21} v^{1}+g_{22} v^{2} \\
v^{1}=g^{11} v_{1}+g^{12} v_{2}, & v^{2}=g^{21} v_{1}+g^{22} v_{2}
\end{array}
$$

where $g^{\alpha \beta}(\alpha, \beta=1,2)$ are the contravariant components of the metric tensor of the bottom surface:

$g^{11}=\frac{g_{22}}{G}, \quad g^{12}=g^{21}=-\frac{g_{12}}{G}, \quad g^{22}=\frac{g_{11}}{G}, \quad G=\operatorname{det}\left(\begin{array}{ll}g_{11} & g_{12} \\ g_{12} & g_{22}\end{array}\right)=g_{11} g_{22}-g_{12}^{2}$. 
In this case, $G \neq 0$ because of the assumption of the absence of singular points on surface (1.7).

Using expressions (1.12), (1.13), one can rewrite equations (1.9), (1.11) in the form of equations for the required functions $v_{\alpha}(\alpha=1,2)$ :

$$
M \frac{\mathrm{d} v_{\alpha}}{\mathrm{d} t}=\frac{M}{2}\left[\left(g_{11}\right)_{q^{\alpha}}\left(v^{1}\right)^{2}+2\left(g_{21}\right)_{q^{\alpha}} v^{1} v^{2}+\left(g_{22}\right)_{q^{\alpha}}\left(v^{2}\right)^{2}\right]+F_{\tau_{\alpha} \sqrt{g_{\alpha \alpha}}} .
$$

Since the landslide is in the rest state at the time moment $t=0$, we have the following initial conditions for system (1.14):

$$
v_{1}(0)=v_{2}(0)=0 \text {. }
$$

Equations (1.14) are supplemented with the system of ordinary differential equations

$$
\frac{\mathrm{d} q^{\alpha}}{\mathrm{d} t}=v^{\alpha}
$$

for determination of the position $\left(q^{1}(t), q^{2}(t)\right)$ of the point $\boldsymbol{x}_{c}(t)$ in the parametric space. In this case the right-hand sides of the latter equations are calculated by formulas (1.13) through the covariant velocity components, and the initial point $\left(q^{1}(0), q^{2}(0)\right)$ is the preimage of the point $\boldsymbol{x}_{c}(0)$ in the parametric space under oneto-one mapping (1.7).

Parameterization (1.7) of the general form is useful in modelling landslide processes in water areas with a complex form of the shore line, for example, in the cases where a shore line cannot be described by a one-value function of the variables $x$ or $y$. To simplify the calculations, we consider further only a simpler case where the parameterization of surface (1.1) can be done with the use of the Cartesian coordinates $x$ and $y$ :

$$
x=x^{1}, \quad y=x^{2}, \quad z=h_{\mathrm{bt}}\left(x^{1}, x^{2}\right) .
$$

The contravariant components $v^{\alpha}$ of the velocity vector coincide with the Cartesian components for this parameterization, therefore, equations (1.14) take the form

$$
M \frac{\mathrm{d} v_{\alpha}}{\mathrm{d} t}=\frac{M}{2}\left[\left(g_{11}\right)_{x^{\alpha}} u^{2}+2\left(g_{12}\right)_{x^{\alpha}} u v+\left(g_{22}\right)_{x^{\alpha}} v^{2}\right]+F_{\tau_{\alpha}} \sqrt{g_{\alpha \alpha}}, \quad \alpha=1,2
$$

in this case $u(0)=0, v(0)=0, v_{1}=g_{11} u+g_{12} v, v_{2}=g_{12} u+g_{22} v$,

$$
g_{11}=1+\left(\frac{\partial h_{\mathrm{bt}}}{\partial x}\right)^{2}, \quad g_{12}=g_{21}=\frac{\partial h_{\mathrm{bt}}}{\partial x} \cdot \frac{\partial h_{\mathrm{bt}}}{\partial y}, \quad g_{22}=1+\left(\frac{\partial h_{\mathrm{bt}}}{\partial y}\right)^{2}
$$

and unit vectors (1.10) tangent to surface (1.1) can be represented as

$$
\boldsymbol{\tau}_{1}=\frac{1}{\sqrt{g_{11}}}\left(1,0, \frac{\partial h_{\mathrm{bt}}}{\partial x}\right)^{T}, \quad \boldsymbol{\tau}_{2}=\frac{1}{\sqrt{g_{22}}}\left(0,1, \frac{\partial h_{\mathrm{bt}}}{\partial y}\right)^{T} .
$$


The Cauchy problem for equation (1.16) is written in parameterization (1.17) in the following way:

$$
\frac{\mathrm{d} x_{c}}{\mathrm{~d} t}=u, \quad \frac{\mathrm{d} y_{c}}{\mathrm{~d} t}=v, \quad x_{c}(0)=x_{c}^{0}, \quad y_{c}(0)=y_{c}^{0}
$$

where $x_{c}^{0}$ and $y_{c}^{0}$ are the known abscissa and ordinate of the point $\boldsymbol{x}_{c}(t)$ at the initial time moment. The third coordinate is determined from the condition (1.3) specifying that the point $\boldsymbol{x}_{c}(t)$ belongs to surface (1.1).

Now let us clarify the way of the calculation of the forces $F_{\tau_{\alpha}}$ from motion equation (1.18) acting on the material point $\boldsymbol{x}_{c}(t)$. In the vertical direction, each elementary volume of the landslide with the cross-section area $\mathrm{d} x \mathrm{~d} y$ is under the action of the gravity and buoyancy forces

$$
\boldsymbol{f}_{g}(x, y)=\left(0,0, f_{g}(x, y)\right)
$$

where $(x, y) \in \mathscr{D}_{t}$,

$$
f_{g}(x, y)=-g\left(\rho_{\mathrm{sl}}-\rho_{w}\right) h_{\mathrm{sl}}(x, y, t) \mathrm{d} x \mathrm{~d} y
$$

$g$ is the acceleration of gravity. Calculate the projections of the force $\boldsymbol{f}_{g}$ onto the directions of the tangent vectors $\tau_{1}$ and $\tau_{2}$ :

$$
f_{g, \tau_{1}}=\boldsymbol{f}_{g} \cdot \boldsymbol{\tau}_{1}=\frac{f_{g}}{\sqrt{g_{11}}} \frac{\partial h_{\mathrm{bt}}}{\partial x}, \quad f_{g, \tau_{2}}=\boldsymbol{f}_{g} \cdot \boldsymbol{\tau}_{2}=\frac{f_{g}}{\sqrt{g_{22}}} \frac{\partial h_{\mathrm{bt}}}{\partial y}
$$

and integrate the obtained expressions over the support $\mathscr{D}_{t}$ of the function $h_{\mathrm{sl}}(x, y, t)$. As the result, we get the contribution of the gravity and buoyancy forces into the components $F_{\tau_{\alpha}}$ :

$$
F_{g, \tau_{\alpha}}(t)=\iint_{\mathscr{D}_{t}} f_{g, \tau_{\alpha}}(x, y, t) \mathrm{d} x \mathrm{~d} y, \quad \alpha=1,2 .
$$

Force (1.22) accelerates the landslide, and its motion slows down due to the water resistance and the landslide friction over the bottom. The resistance force $\boldsymbol{F}_{r}$ is opposite to the landslide motion. By analogy with [20], assume that the magnitude $f_{r}$ of this force is proportional to the greatest area $\Pi$ of the cross-section of the landslide by a vertical plane perpendicular to the vector $(u, v, 0)^{T}$ :

$$
f_{r}=\frac{1}{2} C_{d} \rho_{w} \Pi v_{c}^{2}
$$

where $C_{d}$ is the water resistance coefficient, $v_{c}$ is the absolute value of the vector $\boldsymbol{v}_{c}(t)$ of the motion velocity of the point $\boldsymbol{x}_{c}(t), \quad v_{c}=\left|\boldsymbol{v}_{c}\right|=\left(g_{11} u^{2}+2 g_{12} u v+\right.$ $\left.g_{22} v^{2}\right)^{1 / 2}$. Note that the resistance force $\boldsymbol{F}_{r}$ vanishes for $v_{c}=0$. If the landslide 
moves, the resistance force is determined subject to the motion direction by the formula

$$
\boldsymbol{F}_{r}=-\frac{\boldsymbol{v}_{c}}{v_{c}} f_{r}
$$

and the projections of this force to the directions of the vectors $\tau_{1}$ and $\tau_{2}$ are expressed as

$$
F_{r, \tau_{\alpha}}=\boldsymbol{F}_{r} \cdot \tau_{\alpha}=-\frac{1}{2} \frac{C_{d} \rho_{w} \Pi}{\sqrt{g_{\alpha \alpha}}} v_{c}\left(g_{\alpha 1} u+g_{\alpha 2} v\right)=-\frac{1}{2} \frac{C_{d} \rho_{w} \Pi}{\sqrt{g_{\alpha \alpha}}} v_{c} v_{\alpha}, \quad \alpha=1,2 .
$$

In order to calculate the force of friction, consider again an elementary landslide volume with the cross-section area $\mathrm{d} x \mathrm{~d} y$ and mass $m=\rho_{\mathrm{sl}} h_{\mathrm{sl}}(x, y, t) \mathrm{d} x \mathrm{~d} y$. The force of friction for it is determined according to the normal reaction $N$ acting from the bottom onto the considered element. Calculate $N$ assuming that the elementary volume moves according to equations of form (1.8) written for parameterization (1.17):

$$
m \frac{\mathrm{d} u}{\mathrm{~d} t}=f_{1}, \quad m \frac{\mathrm{d} v}{\mathrm{~d} t}=f_{2}, \quad m \frac{\mathrm{d}}{\mathrm{d} t}\left(u \frac{\partial h_{\mathrm{bt}}}{\partial x}+v \frac{\partial h_{\mathrm{bt}}}{\partial y}\right)=f_{3} .
$$

Multiply the first two equations by $-\partial h_{\mathrm{bt}} / \partial x$ and $-\partial h_{\mathrm{bt}} / \partial y$, respectively, and add the obtained expressions to the third equation. We get

$$
-m \frac{\partial h_{\mathrm{bt}}}{\partial x} \frac{\mathrm{d} u}{\mathrm{~d} t}-m \frac{\partial h_{\mathrm{bt}}}{\partial y} \frac{\mathrm{d} v}{\mathrm{~d} t}+m \frac{\mathrm{d}}{\mathrm{d} t}\left(u \frac{\partial h_{\mathrm{bt}}}{\partial x}+v \frac{\partial h_{\mathrm{bt}}}{\partial y}\right)=f_{n} \sqrt{G}
$$

where $f_{n}=\boldsymbol{f} \cdot \boldsymbol{n}, \boldsymbol{f}=\left(f_{1}, f_{2}, f_{3}\right)^{T}, \boldsymbol{n}=\left(n_{1}, n_{2}, n_{3}\right)^{T}$ is the unit vector normal to surface (1.1),

$$
n_{1}=-\frac{1}{\sqrt{G}} \frac{\partial h_{\mathrm{bt}}}{\partial x}, \quad n_{2}=-\frac{1}{\sqrt{G}} \frac{\partial h_{\mathrm{bt}}}{\partial y}, \quad n_{3}=\frac{1}{\sqrt{G}} .
$$

Applying simple transformations to equation (1.25), we get

$$
\frac{m}{\sqrt{G}}\left(u^{2} \frac{\partial^{2} h_{\mathrm{bt}}}{\partial x^{2}}+2 u v \frac{\partial^{2} h_{\mathrm{bt}}}{\partial x \partial y}+v^{2} \frac{\partial^{2} h_{\mathrm{bt}}}{\partial y^{2}}\right)=f_{n}
$$

The value $f_{n}$ is formed for an elementary landslide volume from the normal component $\boldsymbol{f}_{g} \cdot \boldsymbol{n}$ of force (1.20) and the normal reaction $N$ acting onto this volume. The force of friction is defined as $f_{\mathrm{fr}}=C_{\mathrm{fr}} N=C_{\mathrm{fr}}\left(f_{n}-\boldsymbol{f}_{g} \cdot \boldsymbol{n}\right)$, where $C_{\mathrm{fr}}=\tan \theta_{*}$ is the sliding friction coefficient, $\theta_{*}$ is the angle of friction, $\theta_{*}>0$. Then formulas (1.27), (1.20), (1.21), and (1.26) yield the following expression:

$$
f_{\mathrm{fr}}=\frac{C_{\mathrm{fr}}}{\sqrt{G}}\left[g\left(\rho_{\mathrm{sl}}-\rho_{w}\right)+\rho_{\mathrm{sl}}\left(u^{2} \frac{\partial^{2} h_{\mathrm{bt}}}{\partial x^{2}}+2 u v \frac{\partial^{2} h_{\mathrm{bt}}}{\partial x \partial y}+v^{2} \frac{\partial^{2} h_{\mathrm{bt}}}{\partial y^{2}}\right)\right] h_{\mathrm{sl}}(x, y, t) \mathrm{d} x \mathrm{~d} y
$$


where $u$ and $v$ are the first two components of the vector $\boldsymbol{v}_{c}$. The friction force vector $\boldsymbol{F}_{\mathrm{fr}}(t)$ opposite to the motion is determined by the integration of elementary forces (1.28) over the set $\mathscr{D}_{t}$ :

$$
\boldsymbol{F}_{\mathrm{fr}}(t)=-\frac{\boldsymbol{v}_{c}}{v_{c}} \iint_{\mathscr{D}_{t}} f_{\mathrm{fr}}(x, y, t) \mathrm{d} x \mathrm{~d} y .
$$

Given the force $\boldsymbol{F}_{\text {fr }}$, one can determine its projections onto the directions of the basis vectors $\boldsymbol{\tau}_{1}$ and $\boldsymbol{\tau}_{2}$ :

$$
F_{\mathrm{fr}, \tau_{\alpha}}=\boldsymbol{F}_{\mathrm{fr}} \cdot \boldsymbol{\tau}_{\alpha}=-\frac{v_{\alpha}}{\sqrt{g_{\alpha \alpha}} v_{c}} \iint_{\mathscr{D}_{t}} f_{\mathrm{fr}}(x, y, t) \mathrm{d} x \mathrm{~d} y, \quad \alpha=1,2 .
$$

Note that the calculation of the friction force for $v_{c}=0$ (at the initial time moment and the moments when the landslide stops) is performed by another formula, which we describe below.

The sums of components (1.22), (1.24), (1.29) are taken for the values $F_{\tau_{\alpha}}$ entering the right-hand sides of equations (1.18). Thus, the final form of the landslide motion equations takes the form

$$
\begin{gathered}
\frac{\mathrm{d} v_{\alpha}}{\mathrm{d} t}=\frac{R_{\alpha}}{2}+\left[(\gamma-1) g\left(I_{1, \alpha}-\sigma_{\alpha} C_{\mathrm{fr}} I_{2}\right)-\sigma_{\alpha}\left(\gamma C_{\mathrm{fr}}\left(u^{2} I_{3,11}+2 u v I_{3,12}+v^{2} I_{3,22}\right)\right.\right. \\
\left.\left.+\frac{C_{d}}{2} \Pi v_{c}^{2}\right)\right] \frac{\sqrt{g_{\alpha \alpha}}}{\left(\gamma+C_{w}\right) V}, \quad \alpha=1,2
\end{gathered}
$$

where $\gamma=\rho_{\mathrm{sl}} / \rho_{w}>1, R_{\alpha}=\left(g_{11}\right)_{x^{\alpha}} u^{2}+2\left(g_{12}\right)_{x^{\alpha}} u v+\left(g_{22}\right)_{x^{\alpha}} v^{2}$,

$$
\begin{gathered}
I_{1, \alpha}=-\iint_{\mathscr{D}_{t}} \frac{h_{\mathrm{sl}}(x, y, t)}{\sqrt{g_{\alpha \alpha}(x, y)}} \frac{\partial h_{\mathrm{bt}}}{\partial x^{\alpha}}(x, y) \mathrm{d} x \mathrm{~d} y, \quad I_{2}=\iint_{\mathscr{D}_{t}} \frac{h_{\mathrm{sl}}(x, y, t)}{\sqrt{G(x, y)}} \mathrm{d} x \mathrm{~d} y>0 \\
\sigma_{\alpha}=\frac{v_{\alpha}}{\sqrt{g_{\alpha \alpha} v_{c}}}, \quad I_{3, \alpha \beta}=\iint_{\mathscr{D}_{t}} \frac{h_{\mathrm{sl}}(x, y, t)}{\sqrt{G(x, y)}} \frac{\partial^{2} h_{\mathrm{bt}}}{\partial x^{\alpha} \partial x^{\beta}}(x, y) \mathrm{d} x \mathrm{~d} y, \quad \alpha, \beta=1,2 .
\end{gathered}
$$

Therefore, in order to determine the position of the lower boundary of the liquid, we solve the following problems: using initial data (1.15), we determine the components $v_{\alpha}$ from system of ordinary differential equations (1.30), the Cartesian components $u, v$ of the velocity are calculated by formulas (1.13), problem (1.19) is solved and the coordinates $x_{c}(t), y_{c}(t)$ of the moving point $\boldsymbol{x}_{c}(t)$ are calculated, which allows us to determine the surface of the landslide by formula (1.4) and calculate the lower movable boundary of the fluid at each time moment. All these calculations are performed until the landslide stop, i.e., until the moment when the value $v_{c}$ of the landslide velocity turns to zero.

Note that if the landslide has stopped $\left(v_{c}=0\right)$, this does not mean that it completely stops its motion. For example, a situation is possible where the landslide 
gets a particular speed and moves up the opposite slope of the basin under its own inertia, then stops and begins the backward motion sliding into the deeper part of the water area. Obviously, this continuation of the motion is possible only under a certain proportion between the friction coefficient and the steepness of the slope at the position where the landslide has stopped. Therefore, in order to solve equations (1.30) until the moment of the final landslide stopping, it is necessary to check the criterion of a possible further motion of the landslide after its stop. Let us show what this criterion looks like.

At the stop moment (and also at the initial time moment) the landslide is under the action of two opposite forces whose vectors lie on the plane of the tangent vectors $\boldsymbol{\tau}_{1}$ and $\boldsymbol{\tau}_{2}$, these are the force $\boldsymbol{F}_{g, \tau}$ uniquely determined by its projection (1.22) and the gravity force $\boldsymbol{F}_{\text {fr }}$. The resting landslide begins its motion only under the condition

$$
\left|\boldsymbol{F}_{g, \tau}\right|>\left|\boldsymbol{F}_{\text {fr }}\right|
$$

where

$$
\begin{gathered}
\boldsymbol{F}_{g, \tau}=\frac{F_{g, \tau_{1}}-F_{g, \tau_{2}}\left(\boldsymbol{\tau}_{1} \cdot \boldsymbol{\tau}_{2}\right)}{1-\left(\boldsymbol{\tau}_{1} \cdot \boldsymbol{\tau}_{2}\right)^{2}} \boldsymbol{\tau}_{1}+\frac{F_{g, \tau_{2}}-F_{g, \tau_{1}}\left(\boldsymbol{\tau}_{1} \cdot \boldsymbol{\tau}_{2}\right)}{1-\left(\boldsymbol{\tau}_{1} \cdot \boldsymbol{\tau}_{2}\right)^{2}} \boldsymbol{\tau}_{2} \\
\boldsymbol{F}_{\mathrm{fr}}=-g\left(\rho_{\mathrm{sl}}-\rho_{w}\right) C_{\mathrm{fr}} I_{2} \frac{\boldsymbol{F}_{g, \tau}}{\left|\boldsymbol{F}_{g, \tau}\right|}
\end{gathered}
$$

Taking into account formula (1.22) and equality $\boldsymbol{\tau}_{1} \cdot \boldsymbol{\tau}_{2}=g_{12} / \sqrt{g_{11} g_{22}}$, we get the following expression for criterion (1.31) of the ability of the landslide to move from the rest state:

$$
\left[\frac{g_{11} g_{22}}{G}\left(I_{1,1}^{2}-2 \frac{g_{12}}{\sqrt{g_{11} g_{22}}} I_{1,1} I_{1,2}+I_{1,2}^{2}\right)\right]^{1 / 2}>C_{\mathrm{fr}} I_{2}
$$

which means that the tangent component of the resultant of the gravity and buoyancy forces is greater that the force of friction. It is necessary to check condition (1.32) at the initial time moment too. If it does not hold, then the landslide mass does not move from its position.

\section{Modelling of tsunami-like waves caused by an underwater landslide in a reservoir}

Describe the bottom relief of the model reservoir by the function

$$
z=h_{\mathrm{bt}}(x, y)=h_{\xi}+\left(h_{0}-h_{\xi}\right) \cdot \begin{cases}\left(\frac{x-\xi(y)}{x_{\mathrm{sh}}(y)-\xi(y)}\right)^{2}, & x_{\mathrm{sh}}(y) \leqslant x \leqslant \xi(y) \\ \left(\frac{x-\xi(y)}{L_{x}-\xi(y)}\right)^{2}, & \xi(y) \leqslant x \leqslant L_{x}\end{cases}
$$


where $x \in\left[x_{\mathrm{sh}}(y), L_{x}\right], y \in\left[0, L_{y}\right]$. The 'left' shore (see Fig. 1) is a curvilinear vertical wall forming a cylindrical surface with the directrix parallel to the axis $O z$ and the generatrix given by the equation $x=x_{\mathrm{sh}}(y)$, the 'right' shore is the flat vertical wall $x=L_{x}$. The water depth in the rest state near these vertical walls is $h_{0}<0$, i.e., $h_{0}=h_{\mathrm{bt}}\left(x_{\mathrm{sh}}(y), y\right)=h_{\mathrm{bt}}\left(L_{x}, y\right)=$ const for all $y \in\left[0, L_{y}\right]$. The curve $x=\xi(y)$ is the isobath corresponding to the maximal depth $h_{\xi}=$ const $<h_{0}$ of the basin, in this case $x_{\mathrm{sh}}(y)<\xi(y)<L_{x}$ for all $y$. In each section of surface (2.1) by the plane $y=$ const we get a flat curve composed of the arcs of two parabolas joined smoothly at the bottom point with the abscissa $x=\xi(y)$.

Defining in some way the functions $\xi(y)$ and $x_{\mathrm{sh}}(y)$, we can get different curved bottom surfaces. In this paper these surfaces have been given by the formulas

$$
\begin{aligned}
\xi(y) & =C^{o s}\left(y ; \xi_{1}, \xi_{2}, y_{\xi}, \delta_{\xi}\right) \\
x_{\mathrm{sh}}(y) & =C^{o s}\left(y ; x_{1}, x_{2}, y_{\mathrm{sh}}, \delta_{\mathrm{sh}}\right)
\end{aligned}
$$

where $y_{\xi} \in\left(0, L_{y}\right), \quad \delta_{\xi}>0, \quad y_{\text {sh }} \in\left(0, L_{y}\right), \quad \delta_{\text {sh }}>0$, and the following notation is used:

$C^{o s}\left(q ; p_{1}, p_{2}, q_{0}, \delta\right)= \begin{cases}p_{1}+\frac{p_{2}-p_{1}}{2}\left[1+\cos \left(\frac{2 \pi\left(q-q_{0}\right)}{\delta}\right)\right], & \left|q-q_{0}\right| \leqslant \delta / 2 \\ p_{1}, & \left|q-q_{0}\right|>\delta / 2 .\end{cases}$

The bottom surface presented in Fig. 1 and used in numerical experiments was constructed for the following values of parameters:

$$
\begin{gathered}
h_{0}=-10 \mathrm{~m}, h_{\xi}=-100 \mathrm{~m}, L_{x}=500 \mathrm{~m}, L_{y}=1000 \mathrm{~m}, \xi_{1}=300 \mathrm{~m}, \xi_{2}=150 \mathrm{~m} \\
y_{\xi}=L_{y} / 2, \quad \delta_{\xi}=0.3 L_{y}, x_{1}=100 \mathrm{~m}, x_{2}=0 \mathrm{~m}, y_{\mathrm{sh}}=L_{y} / 2, \quad \delta_{\mathrm{sh}}=0.4 L_{y} .
\end{gathered}
$$

The model water area is characterized by a symmetric 'channel' with smooth slopes in its middle part with respect to the axis $O y$. Assigning zero values to the parameters $x_{1}$ and $x_{2}$ for function (2.3), we get a simpler model water area with a rectilinear 'left' shore. If we additionally require the fulfillment of the equalities $\xi_{1}=\xi_{2}=\xi$, then the form of function (2.2) implies that the shape of the bottom does not depend on the variable $y$. In the latter case for $\xi=L_{x} / 2$ we get the simplest shape of the bottom, which is a cylindrical surface with the generatrix described by the parabolic $\operatorname{arc}$

$$
z=h_{\mathrm{bt}}(x)=\left(h_{0}-h_{\xi}\right)\left(\frac{x}{\xi}-1\right)^{2}+h_{\xi}
$$

and the directrix parallel to the axis $O y$.

Figure 1 shows the trajectories of the landslide motion for two different forms of its initial position. The initial form of the landslide is specified by the formula

$$
h_{\mathrm{sl}}^{0}(x, y)=T \cdot C^{o s}\left(x ; 0,1, x_{c}^{0}, b_{x}\right) \cdot C^{o s}\left(y ; 0,1, y_{c}^{0}, b_{y}\right)
$$




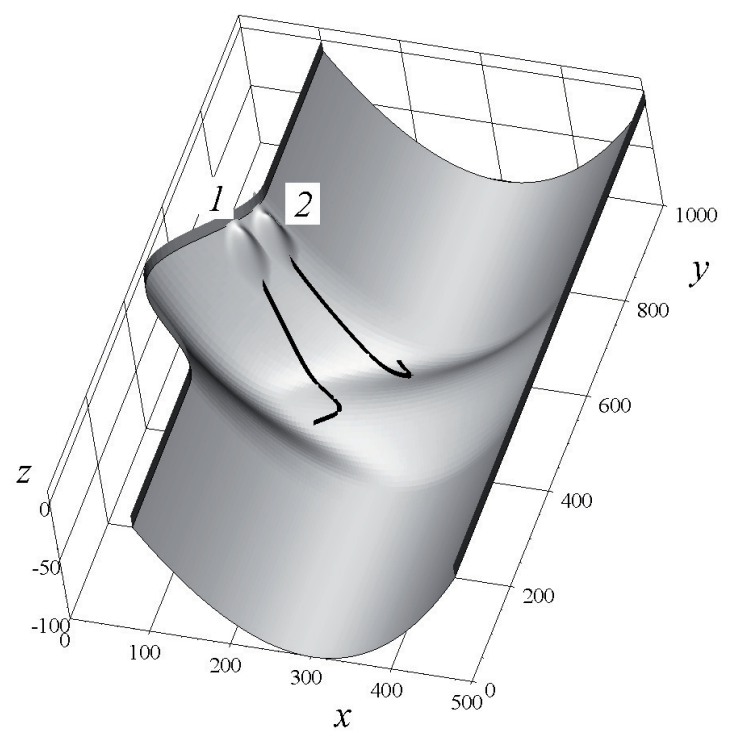

Figure 1. Landslide motion trajectory for two different start positions: $1-x_{c}^{0}=91.5 \mathrm{~m}, y_{c}^{0}=620 \mathrm{~m}$; $2-x_{c}^{0}=115.2 \mathrm{~m}, y_{c}^{0}=660 \mathrm{~m}$.

where $x_{c}^{0}, y_{c}^{0}$ are the given abscissa and ordinate of the top of the landslide for $t=0$, $T$ is the depth of the landslide, $b_{x}, b_{y}$ are its lengths along the axes $O x$ and $O y$, respectively.

The volume of the model landslide is determined by formula (1.6): $V=T b_{x} b_{y} / 4$. According to (1.5), the support of the function $h_{\mathrm{sl}}(x, y, t)$ is the rectangle

$$
\mathscr{D}_{t}=\left[x_{c}(t)-\frac{b_{x}}{2}, x_{c}(t)+\frac{b_{x}}{2}\right] \times\left[y_{c}(t)-\frac{b_{y}}{2}, y_{c}(t)+\frac{b_{y}}{2}\right]
$$

therefore, it is not difficult to calculate the value of $\Pi$ from formula (1.23). It depends on the values of the first two components $u$ and $v$ of the velocity vector $\boldsymbol{v}_{c}(t)$ and is calculated by the following formulas. If one of the components equals zero, then we assume

$$
\Pi=\frac{T}{2} \begin{cases}b_{x}, & u=0, v \neq 0 \\ b_{y}, & u \neq 0, v=0 .\end{cases}
$$

If both components are distinct from zero, then the calculations are performed by the formulas

$$
\Pi=\frac{T}{4} \begin{cases}\sqrt{b_{x}^{2}+\left(k b_{y}\right)^{2}}\left[1+\frac{\sin \pi k}{\pi k\left(1-k^{2}\right)}\right], & |k|<1 \\ \sqrt{\left(b_{x} k_{1}\right)^{2}+b_{y}^{2}}\left[1+\frac{\sin \pi k_{1}}{\pi k_{1}\left(1-k_{1}^{2}\right)}\right], & |k|>1 \\ \frac{3}{2} \sqrt{b_{x}^{2}+b_{y}^{2}}, & |k|=1\end{cases}
$$




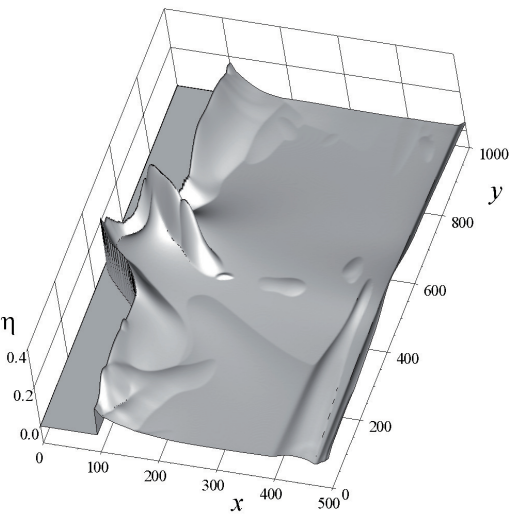

(a)

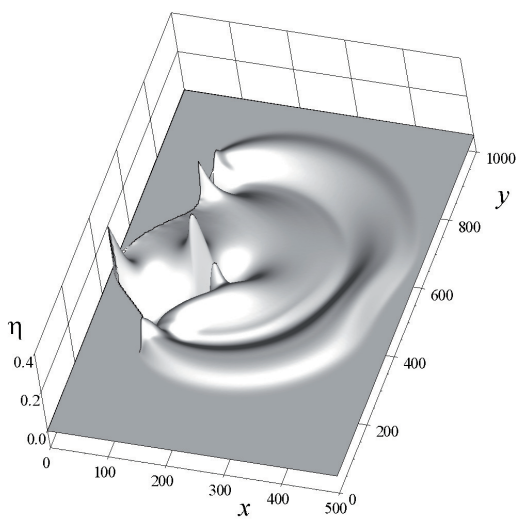

(c)

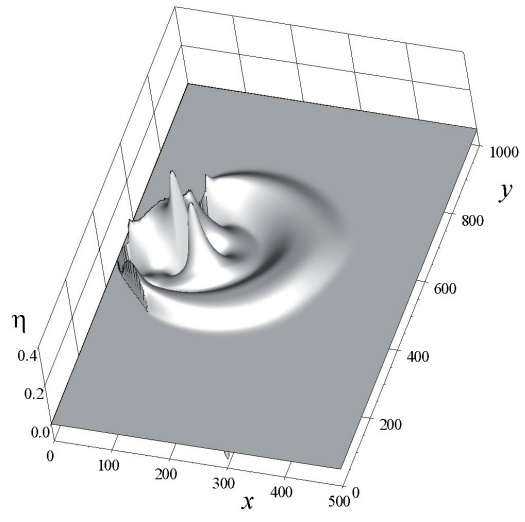

(b)

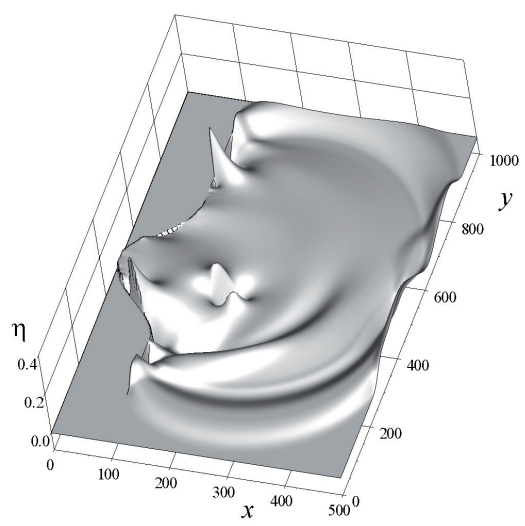

(d)

Figure 2. Graph of maximal amplitude values for the total calculation time (a) and graphs of the free surface $z=\eta(x, y, t)$ at time moments $t=9.9 \mathrm{~s}(\mathrm{~b}), 14.8 \mathrm{~s}(\mathrm{c}), 19.8 \mathrm{~s}(\mathrm{~d})$.

where

$$
k=\frac{u b_{x}}{v b_{y}}, \quad k_{1}=\frac{1}{k}, \quad u \neq 0, v \neq 0 .
$$

In the calculation of the trajectories shown in Fig. 1, the thickness $T$ of the landslide was taken equal to $10 \mathrm{~m}$, the length $b_{x}$ was $50 \mathrm{~m}, b_{y}$ was $50 \mathrm{~m}$. The point $\boldsymbol{x}_{c}(0)$ was given by the coordinates $y_{c}^{0}, z_{c}^{0}=-30 \mathrm{~m}$, and the third one was obtained from the condition that the point belongs to surface (2.1). Motion trajectory $l$ was obtained for $y_{c}^{0}=y_{\mathrm{sh}}+0.3 \delta_{\mathrm{sh}}, 2$ was obtained for $y_{c}^{0}=y_{\mathrm{sh}}+0.4 \delta_{\mathrm{sh}}$, therefore, in the first case we have the value

$$
x_{c}^{0}=\xi\left(y_{c}^{0}\right)+\left(x_{\mathrm{sh}}\left(y_{c}^{0}\right)-\xi\left(y_{c}^{0}\right)\right) \sqrt{\frac{z_{c}^{0}-h_{\xi}}{h_{0}-h_{\xi}}} \approx 91.5 \mathrm{~m}
$$

and in the second case we have the value $x_{c}^{0} \approx 115.2 \mathrm{~m}$. The values of the other 
parameters entering motion law (1.30) were the following: $\gamma=2, C_{w}=1, \theta_{*}=5^{\circ}$, $C_{d}=1$.

Figure 1 shows that the landslide moves from the edge of the channel to increasing depths, then it moves under its own inertia up to a local bottom elevation positioned before the channel, and after that it slides down from this elevation in different directions for two variants 1 and 2 . Varying the governing parameters, we can get other interesting trajectories of the landslide motion, especially for small angles of friction $\theta_{*}$. Thus, the model water area chosen here allows us to consider complex curvilinear motions of landslides and to study waves generated by them.

The general notion of the wave process generated by a landslide motion can be obtained by the glow pattern, which is a spatial distribution of the maximal values of the amplitudes for the whole calculation period (see Fig. 2a). This pattern shows that the main part of the wave energy is directed to the initial movement of the landslide and hence the maximal splashes onto the shore line are observed in this direction. The case of the landslide starting from the point marked by digit 1 in Fig. 1 was numerically simulated for the critical parameters indicated above with the use of the MacCormac scheme on a uniform grid within the systematic nonlinear shallow water model. The nonpercolation condition was posed on the 'left', $x=x_{\mathrm{sh}}(y)$ and 'right', $x=L_{x}$ shores of the reservoir, the condition of free wave passage was posed on the other two boundaries $y=0$ and $y=L_{y}$.

Figures $2 b-2 d$ demonstrate the evolution of the surface wave process. It is seen that at the initial moment of the landslide movement a positive wave is generated, this wave spreads in all directions and passes ahead of the landslide moving at a subcritical velocity. A negative escort wave is observed over the landslide during its total motion, two positive 'splashes' are also observed over its front and back. In contrast to the leading positive wave mentioned above, which does not depend on the landslide and its motion characteristics except for the moment of its generation, the amplitudes of these 'escorts' are completely determined by the landslide motion velocity and the depth of its current position. The smaller the velocity and the greater the depth, the lower the height of those waves and vise versa. As the waves spread, they start interacting with the bottom unevenness and the shore lines.

\section{3. 'One-dimensional' landslide motion equation}

A complicated pattern of the wave modes in a landslide motion over a spatially uneven slope of a reservoir and the difficulties in interpreting the results obtained under varying a large number of parameters make the study of wave generation necessary and useful even in the simpler case where the form of the landslide and the bottom configuration do not depend on one of the horizontal coordinates.

Thus, let bottom relief (1.1) and form of landslide (1.4) not depend, for instance, on the coordinate $y$ and be described by the functions

$$
\begin{aligned}
& z=h_{\mathrm{bt}}(x) \\
& z=h_{\mathrm{sl}}(x, t)=h_{\mathrm{sl}}^{0}\left(x+x_{c}^{0}-x_{c}(t)\right) .
\end{aligned}
$$


Then the landslide moves parallel to the plane $x O z(v \equiv 0)$ and the following equalities hold:

$$
\begin{gathered}
\frac{\partial h_{\mathrm{bt}}}{\partial y} \equiv 0, \quad g_{12}=g_{21} \equiv 0, \quad g_{22} \equiv 1, \quad G=g_{11} \\
v_{1}=g_{11} u, \quad v_{c}=\sqrt{g_{11}}|u|, \quad \Pi=T b_{y}
\end{gathered}
$$

where $T$ is the maximal landslide thickness in the vertical direction. The motion of the landslide is described by the first equation of (1.30), which takes the following simpler form in the case considered here:

$$
\frac{\mathrm{d}}{\mathrm{d} t}\left(g_{11} u\right)=\frac{R}{2}+\left[(\gamma-1) g\left(I_{1}-\sigma C_{\mathrm{fr}} I_{2}\right)-\sigma\left(\gamma C_{\mathrm{fr}} I_{3} u^{2}+\frac{C_{d}}{2} T b_{y} v_{c}^{2}\right)\right] \frac{\sqrt{g_{11}}}{\left(\gamma+C_{w}\right) V}
$$

where $\sigma=\operatorname{sign} u, R=g_{11}^{\prime} u^{2}, g_{11}^{\prime}=\mathrm{d} g_{11} / \mathrm{d} x, g_{11}=1+\left(h_{\mathrm{bt}}^{\prime}\right)^{2}, h_{\mathrm{bt}}^{\prime}=\mathrm{d} h_{\mathrm{bt}} / \mathrm{d} x$, and for the sake of brevity, the values $I_{1,1}$ and $I_{3,11}$ are redenoted by $I_{1}$ and $I_{3}$.

The independence of functions (3.2) of the variable $y$ leads to a particular form of the sets $\mathscr{D}_{0}$ and $\mathscr{D}_{t}$ :

$$
\mathscr{D}_{0}=\left[x_{l}(0), x_{r}(0)\right] \times\left[y_{l}, y_{r}\right], \quad \mathscr{D}_{t}=\left[x_{l}(t), x_{r}(t)\right] \times\left[y_{l}, y_{r}\right]
$$

and for all $t \geqslant 0$ the following conditions hold: $x_{c}(t) \in\left(x_{l}(t), x_{r}(t)\right), y_{c}(t) \equiv y_{c}^{0} \in$ $\left(y_{l}, y_{r}\right), \quad y_{r}=y_{l}+b_{y}, \quad x_{l}(t)=x_{l}(0)+x_{c}(t)-x_{c}^{0}, \quad x_{r}(t)=x_{l}(t)+b_{x}$. Thus, in the case of 'one-dimensional' motion of the landslide considered here its volume (1.6) is determined by the formula $V=b_{y} S_{0}$, where $S_{0}$ is the cross-section of the landslide by a plane perpendicular to the axis $O y$ and this section does not depend on $y$,

$$
S_{0}=\int_{x_{l}(t)}^{x_{r}(t)} h_{\mathrm{sl}}(x, t) \mathrm{d} x=\int_{x_{l}(t)}^{x_{r}(t)} h_{\mathrm{sl}}^{0}\left(x+x_{c}^{0}-x_{c}(t)\right) \mathrm{d} x=\int_{x_{l}(0)}^{x_{r}(0)} h_{\mathrm{sl}}^{0}(x) \mathrm{d} x=\text { const. }
$$

The integrals in equation (3.3) can be represented as

$$
\begin{gathered}
I_{1}=b_{y} \int_{x_{l}(t)}^{x_{r}(t)} h_{\mathrm{sl}}(x, t) \sin \theta(x) \mathrm{d} x, \quad I_{2}=b_{y} \int_{x_{l}(t)}^{x_{r}(t)} h_{\mathrm{sl}}(x, t) \cos \theta(x) \mathrm{d} x \\
I_{3}=b_{y} \int_{x_{l}(t)}^{x_{r}(t)} h_{\mathrm{sl}}(x, t) \frac{h_{\mathrm{bt}}^{\prime \prime}(x)}{\sqrt{g_{11}}} \mathrm{~d} x,
\end{gathered}
$$

where $\theta(x)=-\arctan h_{\mathrm{bt}}^{\prime}(x)$ is local bottom inclination angle (3.1),

$$
\tan \theta(x)=-h_{\mathrm{bt}}^{\prime}(x), \quad \sin \theta(x)=-\frac{h_{\mathrm{bt}}^{\prime}(x)}{\sqrt{g_{11}}}, \quad \cos \theta(x)=\frac{1}{\sqrt{g_{11}}} .
$$


The formulas presented here imply that the motion law of the point $\boldsymbol{x}_{c}(t)$ does not depend on the width of the landslide $b_{y}$.

From nonlinear ordinary differential equation (3.3) with the use of the initial conditions

$$
x_{c}(0)=x_{c}^{0}, \quad u(0)=0
$$

taking into account the notation $u=\dot{x}_{c}$, we can calculate the abscissa $x_{c}(t)$ of the moving point $\boldsymbol{x}_{c}(t)$ and thus obtain the surface of the landslide from formula (3.2) and determine the lower moving boundary of the fluid at each time moment.

It is not difficult to check that for the flat slope

$$
z=h_{\mathrm{bt}}(x)=h_{0}-x \tan \theta, \quad x \geqslant 0, \quad \theta=\text { const }
$$

equation (3.3) is written in the form of the equation given in [11]. Moreover, in the case of a flat slope one can easily write the exact solution to problem (3.3), (3.4).

For a curvilinear bottom profile, the solution to problem (3.3), (3.4) can be calculated with the use of numerical integration methods. Figure 3 a shows the abscissa (line 3) of the top of the landslide having the initial form

$$
h_{\mathrm{sl}}^{0}(x, y)=T \cdot C^{o s}\left(x ; 0,1, x_{c}^{0}, b_{x}\right)
$$

and moving over an uneven bottom with the profile shaped as a parabolic arc (2.4). In these calculations we used the following values of the parameters responsible for the bottom form: $h_{\xi}=-100 \mathrm{~m}, h_{0}=-10 \mathrm{~m}, \xi=250 \mathrm{~m}$. On the sides (at the points $x=0$ and $x=500$ ), the reservoir is bounded by vertical impermeable walls. At the initial time moment the top of the landslide is above the bottom point corresponding to the depth $z_{c}^{0}=-30 \mathrm{~m}$, therefore,

$$
x_{c}^{0}=\xi\left(1-\sqrt{\frac{z_{c}^{0}-h_{\xi}}{h_{0}-h_{\xi}}}\right) \approx 29.5 \mathrm{~m} .
$$

The values of the parameters of motion equation (3.3) were the following:

$$
T=10 \mathrm{~m}, \quad b_{x}=50 \mathrm{~m}, \quad \gamma=2, \quad C_{w}=1, \quad \theta_{*}=5^{\circ}, \quad C_{d}=1 .
$$

It is worth noting that landslide (3.2), (3.5) satisfies the formula $S_{0}=T b / 2$ and the value $T$ is a linear multiplier in the integrals $I_{1}, I_{2}, I_{3}$ from equation (3.3), therefore, it is canceled and the motion law of the landslide does not change under a variation in its thickness $T$.

The analysis of the graph of the function $x_{c}(t)$ (line 3 in Fig. 3a) shows that for these values of the parameters the landslide goes over the point $x=\xi$ of the greatest depth and moves up the opposite slope under its own inertia, then it stops and moves back a little and finally stops slightly lower than the point of its first stop. More complicated landslide motion trajectories with several changes of the motion direction may occur for a small coefficient of friction (see Fig. 3a, line 4 


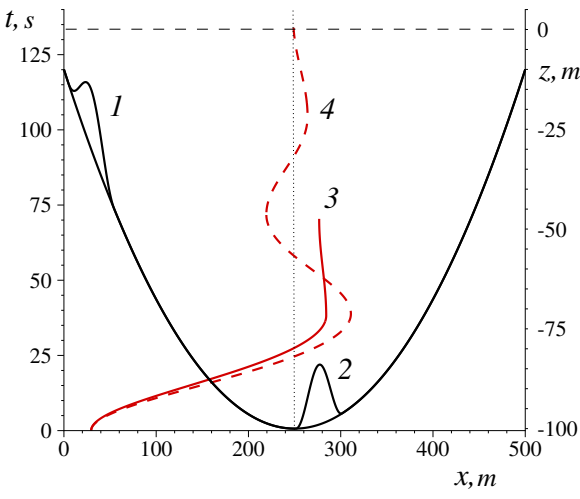

(a)

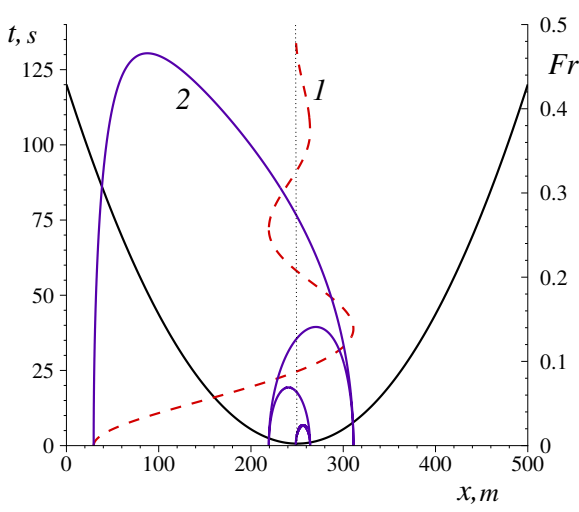

(b)

Figure 3. Landslide motion over an uneven bottom of the model reservoir. (a): 1, 2 form of landslide at the initial time moment (1) and at the stop moment for $\theta_{*}=5^{\circ}(2) ; 3,4$ graphs of the function $x=x_{c}(t)$ for $\theta_{*}=5^{\circ}(3)$ and $\theta_{*}=1^{\circ}(4)$; (b): graphs of the function $x=x_{c}(t)(1)$ and the dependence of the Froude number $F r$ on the coordinate $x_{c}(t)$ (2) for $\theta_{*}=1^{\circ}$.

corresponding to $\theta_{*}=1^{\circ}$ ). Nevertheless, even for very small values of the coefficient of friction, the motion of the landslide over bottom (2.4) proceeds at subcritical velocities (see line 2 in Fig. 3b representing the dependence of the local Froude number $F r=v_{c}\left(x_{c}\right) / \sqrt{g h_{\mathrm{bt}}\left(x_{c}\right)}$ of the abscissa $x_{c}(t)$ of the moving point $\boldsymbol{x}_{c}(t)$ ). Recall also that we should check stopping criterion (1.32) at each stop moment, and in the one-dimensional case this criterion takes the form of the inequality

$$
\left|I_{1}\right|>C_{\mathrm{fr}} I_{2} .
$$

Condition (3.7) should be checked at the initial time moment too. The landslide mass with initial form (3.5) lying on uneven bottom (2.4) does not leave its place for the values of $b_{x}$ and $z_{c}^{0}$ indicated above if $\theta_{*} \geqslant 32.5^{\circ}$.

\section{Some results of calculations}

Numerical simulation of surface waves generated by landslide motion (3.5) over a slope of parabolic form (2.4) has been performed within the nonlinear shallow water model using a predictor-corrector scheme of the second order of approximation [17] on an adaptive grid. The grid was constructed by the method of equidistribution [14] with the use of a control function tracing movable elevations and troughs of the waves.

For a bounded reservoir we have the following wave pattern generated by a landslide. A solitary wave is gradually formed on the water surface before the speedingup landslide. This wave moves toward increasing depth at a speed exceeding that of the landslide. Just over the moving landslide the free boundary has the form of a negative wave, i.e., a 'trough' going away from the shore together with the landslide (see Fig. 4a). The front rise wave is reflected from the opposite shore and goes 


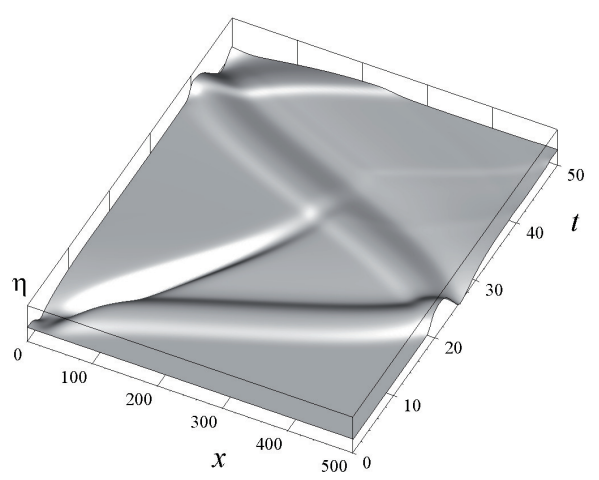

(a)

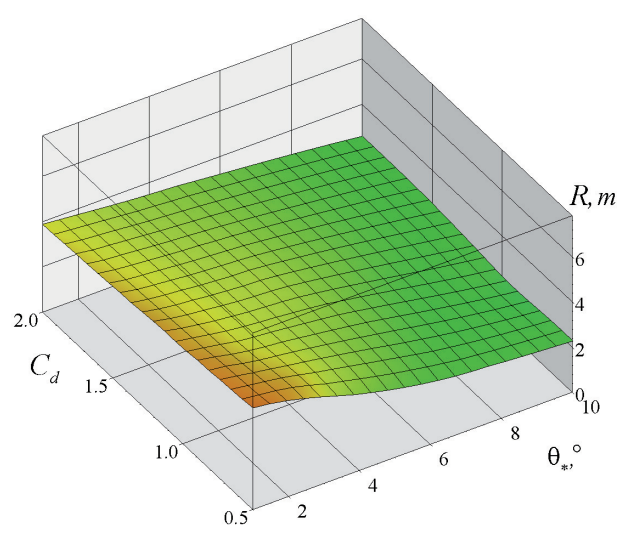

(c)

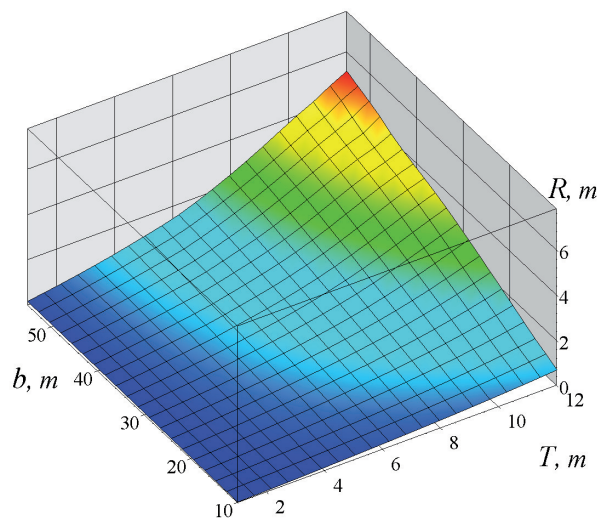

(b)

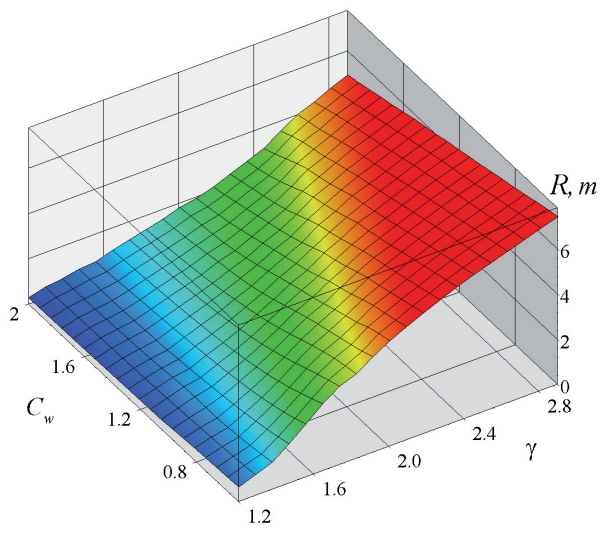

(d)

Figure 4. Graph of the surface $z=\eta(x, t)$ (a) and graphs of dependences of the maximal splashing $R$ on the length $b$ and height $T$ of the underwater landslide (b), on the resistance coefficient $C_{d}$ and the angle of friction $\theta_{*}$ (c), on the coefficient of associated mass $C_{w}$ and relative density of landslide mass $\gamma(\mathrm{d})$.

through the reservoir in the reverse direction. It interacts with the 'trough' and then goes onto the waterside slope from which the landslide has slid. A solitary wave of a considerable amplitude is formed after the reflections from the opposite shores, such wave may pass across the reservoir many times. In this case, for parabolic bottom (2.4) we get similar values of the maximal splashes on the opposite shores of the reservoir.

Below we present the results of studies clarifying the rate of influence of the parameters $T, b=b_{x}, \gamma, C_{w}, \theta_{*}, C_{d}$ on the value $R$ of the maximal splashing onto the 'right' shore of the reservoir $(x=500 \mathrm{~m})$. In this case we varied the values of a pair of parameters and fixed the values of the other parameters taken as in (3.6); in all experiments we used the constant values of $h_{\xi}, h_{0}, \xi$, and $z_{c}^{0}$ indicated above. 
Figure $4 \mathrm{~b}$ shows the surface $R=R(b, T)$ characterizing the effect of the landslide size $(b=10 \div 55 \mathrm{~m}, T=1 \div 12 \mathrm{~m})$ and hence its volume $V$ on the maximal splash. We have indicated above that the model landslide considered here has the motion law not dependent on the value of $T$. However, the amplitude of the generated waves essentially depends on the parameter $T$, it grows with an increasing landslide thickness. A variation of $b$ for the constant values (3.6) of the other parameters affects the motion law so that a longer landslide moves at a greater speed and thus generates stronger surface waves. Therefore, an increase in the length $b$ of the landslide causes the same qualitative changes in the splashing values as an increase in its thickness $T$. Note that the critical effect on the value of $R$ is caused by the motion of the landslide in the shallow part of the water area, whereas in the descent of the landslide into the zone of large depths, the energy transmission from the moving landslide to the surface waves practically stops. Since the influence of the landslide volume on the amplitude of the generated waves becomes apparent from the very beginning of the motion even in the shallow part and the speed of its motion increases with the growth of the volume, the size of the landslide is an important determinant factor in the estimation of the splashing values on the shores of the reservoir.

Figure $4 \mathrm{c}$ demonstrates the dependence of the maximal splash $R$ on the parameters responsible for deceleration of the landslide that are difficult to determine, i.e., the coefficient of friction $C_{d}$ and the angle of friction $\theta_{*}$. It can be seen that for a sufficiently wide range of these parameters $\left(C_{d}=0.5 \div 2, \theta_{*}=1 \div 10^{\circ}\right)$ their influence on $R$ is weaker than the variation of the volume. The analysis of landslide motion trajectories shows that the route and the velocity of the landslide decrease with an increase of friction. For small $\theta_{*}$ the velocity of the landslide is greater, but it goes faster into the depth where its movement has little effect on the process of wave generation, as was indicated above. As seen from Fig.4c, the variation of the hydrodynamic resistance coefficient $C_{d}$ has an even weaker influence on the splashing values. This relates to the fact that the variation of this coefficient is significant for large landslide velocities, and the latter are realized in the zone of large depths.

It is seen from Fig. $4 \mathrm{~d}$ that among the two parameters $C_{w}$ and $\gamma$ characterizing the moving landslide mass, the variation of the first one $\left(C_{w}=0.5 \div 2\right)$ slightly affects the maximal splash $R$, but the second one $(\gamma=1.2 \div 2.9)$ has an essential influence. The latter relates to the fact that a landslide having a larger relative density can get a considerable speed even in the shallow part of the water area, which results in the generation of high surface waves.

Summarizing, we can say that among all parameters of the landslide motion equation (3.3) the most essential influence on the maximal splash values is caused by the landslide size and its density, i.e., the parameters easily estimated in field measurements. Large volumes of the landslide mass moving in shallow zones generate high surface waves producing big splashes and flooding the shore. 


\section{Conclusion}

In this paper we derive motion equations for a 'quasi-rigid' landslide moving over an uneven spatially inhomogeneous underwater slope and, considering the shallow water model, study numerically the influence of the parameters of the motion law on the maximal shore splashing of surface waves generated by a landslide in the case of a bounded reservoir. Thus, we show that among all parameters considered in the paper, the size and density of the landslide cause the main effect on the maximal splash value, i.e., these are the parameters which can be estimated in field studies with a sufficient accuracy. At the same time, such parameters as the coefficients of associated mass and hydrodynamic resistance and also the angle of friction that are hard to estimate do not practically affect the value of splashing on the shore for a wide range of variations.

It is worth noting that dangerous underwater landslides are the most probable only in deep reservoirs with steep waterside slopes. Therefore, the shallow water theory in its long-wave approximation can give only approximate estimates of splashing for such reservoirs. Test calculations based on the complete equations of wave hydrodynamics show that a landslide generates not just one, but a series of comparatively short surface waves moving to the shore at a lower speed than the waves in the nonlinear shallow water model and hence give lower values of maximal splashing. Thus, the use of the shallow water model of the first approximation leads to overestimation of the maximal splashing onto the shores of a deep bounded reservoir and requires taking into account the dispersive effects, in order to obtain a more accurate estimate. In this context, we plan to undertake a multiparametric study of surface waves generated by a landslide within nonlinear-dispersive models of wave hydrodynamics $[3,7,8,16]$.

\section{References}

1. J.-P. Bardet, C. E. Synolakis, H. L. Davies, F. Imamura, and E. A. Okal, Landslide tsunamis: recent findings and research directions. Pure Appl. Geophys. (2003) 160, 1793-1809.

2. S. A. Beizel, L. B. Chubarov, and G. S. Khakimzyanov, Simulation of surface waves generated by an underwater landslide moving over an uneven slope. Russ. J. Numer. Anal. Math. Modelling (2011) 26, No. 1, 17-38.

3. L. B. Chubarov, S. V. Eletskij, Z. I. Fedotova, and G. S. Khakimzyanov, Simulation of surface waves generation by an underwater landslide. Russ. J. Numer. Anal. Math. Modelling (2005) 20 , No. 5, 425-437.

4. L. B. Chubarov, G. S. Khakimzyanov, and N. Yu. Shokina, Numerical modelling of surface water waves arising due to movement of underwater landslide on irregular bottom slope. In: Notes on Numerical Fluid Mechanics and Multidisciplinary Design. Computational Science and High Performance Computing IV, Vol. 115, Springer-Verlag, Berlin-Heidelberg, 2011, pp. 75-91.

5. I. Didenkulova, I. Nikolkina, E. Pelinovsky, and N. Zahibo, Tsunami waves generated by submarine landslides of variable volume: analytical solutions for a basin of variable depth. Natural Hazards Earth System Sci. (2010) 10, No. 11, 2407-2419.

6. M. Di Risio, G. Bellotti, A. Panizzo, and P. De Girolamo, Three-dimensional experiments on landslide generated waves at a sloping coast. Coastal Engrg. (2009) 56, 659-671. 
7. D. Dutykh, T. Katsaounis, and D. Mitsotakis, Finite volume schemes for dispersive wave propagation and runup. J. Comput. Phys. (2011) 230, 3035-3061.

8. D. Dutykh, D. Mitsotakis, S. Beysel, and N. Shokina, Dispersive waves generated by an underwater landslide. In: Numerical Methods for Hyperbolic Equations: Theory and Applications. Int. Conf. to honour Professor E. F. Toro in the month of his 65th birthday, 2011 (in print).

9. S. V. Eletskii, Yu. B. Maiorov, V. V. Maksimov, I. S. Nudner, Z. I. Fedotova, M. G. Khazhoyan, G. S. Khakimzyanov, and L. B. Chubarov, Simulation of surface waves generation by a moving part of the bottom down the coastal slope. Comp. Tech. (2004) 9, Part 2, 194-206.

10. F. Enet and S. T. Grilli, Experimental study of tsunami generation by three-dimensional rigid underwater landslides. J. Waterway Port Coastal Ocean Eng. (2007) 133, No. 6, 442-454.

11. S. T. Grilli and P. Watts, Tsunami generation by submarine mass failure. I : Modelling, experimental validation, and sensitivity analyses. J. Waterway Port Coastal Ocean Eng. (2005) 131, No. 6, 283-297.

12. C. B. Harbitz, G. Pedersen, and B. Gjevik, Numerical simulation of large water waves due to landslides. J. Hydraulic Eng. (1993) 119, 1325-1342.

13. C. B. Harbitz, F. Lovholt, G. Pedersen, S. Glimsdal, and D. G. Masson, Mechanisms of tsunami generation by submarine landslides - a short review. Norwegian J. Geology (2006) 86, No. 3, $255-264$.

14. G. S. Khakimzyanov, Yu. I. Shokin, V. B. Barakhnin, and N. Yu. Shokina, Numerical Simulation of Fluid Flows with Surface Waves. Sib. Branch, Russ. Acad. Sci., Novosibirsk, 2001 (in Russian).

15. E. Pelinovsky and A. Poplavsky, Simplified model of tsunami generation by submarine landslides. J. Phys. Chem. Earth (1996) 21, No. 12, 13-17.

16. Yu. I. Shokin, Z. I. Fedotova, G. S. Khakimzyanov, L. B. Chubarov, and S. A. Beizel, Modelling surfaces waves generated by a moving landslide with allowance for vertical flow structure. Russ. J. Numer. Anal. Math. Modelling (2007) 22, No. 1, 63-85.

17. Yu. I. Shokin, Yu. V. Sergeeva, and G. S. Khakimzyanov, Predictor-corrector scheme for the solution of shallow water equations. Russ. J. Numer. Anal. Math. Modelling (2006) 21, No. 5, $459-479$.

18. S. Tinti, E. Bortolucci, and C. Vannini, A block-based theoretical model suited to gravitational sliding. Natural Hazards (1997) 16, 1-28.

19. P. Watts, S. T. Grilli, J. T. Kirby, G. J. Fryer, and D.R. Tappin, Landslide tsunami case studies using a Boussinesq model and a fully nonlinear tsunami generation model. Natural Hazards Earth System Sci. (2003) 3, No. 5, 391-402.

20. P. Watts, F. Imamura, and S. T. Grilli, Comparing model simulations of three benchmark tsunami generation cases. Sci. Tsunami Hazards (2000) 18, No. 2, 107-123. 